\title{
Consequências neurológicas causadas pela SARS-CoV-2
}

\author{
Neurological consequences caused by SARS-CoV-2 \\ Consecuencias neurológicas causadas por SARS-CoV-2
}

\author{
Maria Gabryelle da Silva Soares \\ ORCID: https://orcid.org/0000-0003-2575-8267 \\ Universidade Federal de Campina Grande, Brasil \\ E-mail: gabryelle8685@gmail.com \\ Maria Letícia Cardoso da Silva Barbosa \\ ORCID: https://orcid.org/0000-0002-2935-6882 \\ Universidade Federal de Campina Grande, Brasil \\ E-mail: marialeticia20151@ hotmail.com \\ Maria Eduarda Wanderley de Barros Silva \\ ORCID: https://orcid.org/0000-0002-4642-3282 \\ Universidade Federal de Campina Grande, Brasil \\ E-mail: eduarda.wanderley@outlook.com \\ Kaliana Xavier Siqueira \\ ORCID: https://orcid.org/ 0000-0002-7138-5740 \\ Universidade Federal de Campina Grande, Brasil \\ E-mail: kalianaxavier90@gmail.com \\ Ana Regina da Silva Pereira \\ ORCID: https://orcid.org/0000-0001-9213-0686 \\ Universidade Federal de Campina Grande, Brasil \\ E-mail: anaregiina_@outlook.com \\ Bianca Maria Mendes da Silva \\ ORCID: https://orcid.org/0000-0002-2311-3345 \\ Universidade Federal de Pernambuco, Brasil \\ E-mail: bianca.mendes@ufpe.br \\ Rodrigo Medeiros Gurgel de Faria \\ ORCID: https://orcid.org/0000-0003-4001-0783 \\ Universidade Potiguar, Brasil \\ E-mail: rodrigomgf95@gmail.com \\ Arthu Línniker Lopes de Oliveira \\ ORCID: https://orcid.org/0000-0002-8882-392X \\ Universidade Potiguar, Brasil \\ E-mail: arthulinniker@gmail.com \\ Thalita Rafael do Nascimento \\ ORCID: https://orcid.org/0000-0002-4915-4815 \\ Universidade Potiguar, Brasil \\ E-mail: thalitarafael.12@gmail.com \\ Isis Yohana Garcia Cipriano \\ ORCID: https://orcid.org/0000-0001-7433-014X \\ Universidade Potiguar, Brasil \\ E-mail: isisyohanaa@gmail.com \\ Ednilma Barbosa Silva \\ ORCID: https://orcid.org/0000-0002-4327-5579 \\ Universidade Federal do Rio Grande do Norte, Brasil \\ E-mail: ednilmabsilva@gmail.com \\ Renata da Silva Ferreira \\ ORCID: https://orcid.org/0000-0002-9614-6930 \\ Centro Universitário Maurício de Nassau, Brasil \\ E-mail: renataodilon21@gmail.com \\ Larissa Lima Soares \\ ORCID: https://orcid.org/0000-0002-7682-372X \\ Centro Universitário Tiradentes, Brasil \\ E-mail: larissalyma@hotmail.com
}

\section{Resumo}

Com a crise do novo coronavírus se tem diversas consequências a longo prazo para sociedade visto que centenas de países vivem em regime de distanciamento físico e isolamento social. Estudos comprovam também a existência de sintomas neurológicos em pacientes com COVID-19, tais como cefaleia, tontura e consciência prejudicada como o destaque, para as células do sistema nervoso central que podem ser infectadas juntamente com sistema respiratório. 
Trata-se de uma revisão integrativa de literatura, realizada conforme as etapas: elaboração da questão de pesquisa, levantamento bibliográfico, avaliação dos dados, análise dos dados e apresentação dos resultados. As bases de dados utilizadas foram Medical Literature Analysis and Retrieval System Online e Science Direct. A busca nas bases de dados resultou em 1.768 publicações, as quais foram selecionadas pelos critérios de elegibilidade, resultando na amostra final de 12 artigos. Foram identificados diferentes danos neurológicos associados a infecção por COVID-19, os quais possibilitam discussões sobre como essas consequências podem acontecer e quais os fatores envolvidos. Considera-se que os danos graves, estão mais presentes na população de grupo de risco, idosos e pessoas com comorbidades. Os estudos associados ás consequências da SARS-CoV-2 estão bastante direcionados aos cuidados e atenção ao sistema respiratório, o que promove um número mais reduzido de estudos direcionados ao sistema neurológico, contudo, há evidências, séries de casos e estudos que representam danos neurológicos pelo novo coronavírus. Assim, as manifestações neurológicas encontradas em pacientes com COVID-19 necessitam de assistência para auxiliar os provedores no tratamento, gerenciamento e reconhecimento nas complicações neurológica potencialmente fatais.

Palavras-chave: COVID-19; Doenças do Sistema Nervoso; SARS-CoV-2.

\begin{abstract}
The crisis of the new coronavirus has several long-term consequences for society as hundreds of countries live in a regime of physical distance and social isolation. Studies also prove the existence of neurological symptoms in patients with COVID-19, such as headache, dizziness and impaired consciousness. This is an integrative literature review, carried out according to the steps: elaboration of the research question, bibliographic survey, data evaluation, data analysis and presentation of results. The databases used were Medical Literature Analysisand Retrieval System Online and Science Direct. The search in the databases resulted in 1,768 publications, which were selected by the eligibility criteria, resulting in a final sample of 12 articles. Different neurological damages associated with COVID-19 infection were identified, which allow discussions on how these consequences can happen and what factors are involved. It is considered that serious damage is more present in the population of risk groups, the elderly and people with comorbidities. The studies associated with the consequences of SARS-CoV-2 are very much directed to care and attention to the respiratory system, which promotes a smaller number of studies directed to the neurological system, however, there is evidence, case series and studies that represent neurological damage by the new coronavirus. Thus, the neurological manifestations found in patients with COVID-19 need assistance to assist providers in the treatment, management and recognition of potentially fatal neurological complications.
\end{abstract}

Keywords: COVID-19; Nervous System Diseases; SARS-CoV-2.

\title{
Resumen
}

La crisis del nuevo coronavirus tiene varis consecuencias a largo plazo para la sociedad, ya que cientos de países viven um régimen de distancia física y aislamiento social. Los estúdios también muestran la existencia de síntomas neurológicos en pacientes con COVID-19, como Dolores de cabeza, mareos y deterioro de la conciencia. Es una revisión integradora de la literatura, realizada según los pasos: elaboración de la pregunta de investigación, relevamiento bibliográfico, evaluación de datos, análisis de datos y presentación de resultados. Las bases de datos utilizadas fueron Medical Literature Analysis and Retrieval System Online y Science Direct. La búsqueda en las bases de datos arrojó 1.768 publicaciones, lãs cuales fueron seleccionadas por los criterios de elegibilidad, dando como resultado una muestra final de 12 artículos. Se identificaron diferentes daños neurológicos asociados a la infección por COVID-19, lo que permiten discutir cómo puedeno ocurrir estas consecuencias y qué factores están involucrados. Se considera que el daño severo está más presente em la población de grupos de riesgo, ancianos y personas concomorbilidades. Los estúdios asociados a lãs consecuencias del SARS-CoV-2 están muy enfocados al cuidado y atención al sistema respiratorio, lo que promueve un menor número de estudios dirigidos al sistema neurológico, sin embargo, existen evidencias, series de casos y estudios que representan daño neurológico por el nuevo coronavirus. Por lo tanto, lãs manifestaciones neurológicas encontradas en pacientes con COVID-19 necesitan asistencia para ayudar a los proveedores em el tratamiento, manejo y reconocimiento de complicaciones neurológicas potencialmente fatales.

Palabras clave: COVID-19; Enfermedades del Sistema Nervioso; SARS-CoV-2.

\section{Introdução}

A Organização Mundial de Saúde (OMS), decretou pandemia global do covid-19 com os primeiros casos de uma síndrome gripal com evolução para a Síndrome Aguda Respiratória na cidade de Wuhan, na China, doença causada pelo novo coronavírus (Sars-CoV-2). Os fatores de risco para um pior prognóstico incluem comorbidades como diabetes, hipertensão, câncer e idade avançada, tendo como taxa de letalidade a depender das políticas de prevenção e controle implementadas, 
capacidade do sistema de saúde de cada país e abrangência das testagens tendendo assim a aumentar em localidade de baixa renda e sem acesso a instalações de cuidados hospitalares (Couto, Barbieri \& Matos., 2021).

Com isso, o mundo vem acompanhando um quadro sanitário sem precedentes nos últimos 100 anos, com 15 milhões de casos confirmados e 640 mil mortes a menos de cinco meses desde o decreto da pandemia pela OMS. Para que se tenha a redução de novos casos foram implementadas medidas de controle para promover o achatamento da curva epidêmica incluindo assim quarentena aos expostos, distanciamento físico, isolamento social e orientações sobre o uso de máscaras visando diminuir assim a circulação do vírus (OPAS., 2020).

Com a crise do novo coronavírus se tem diversas consequências a longo prazo para sociedade visto que centenas de países vivem em regime de distanciamento físico e isolamento social com o fechamento de escolas, comércios e indústrias com o intuito de reduzir a propagação do vírus implicando assim recessão econômica. Para que se tenha a redução dessas consequências é preciso que se tenha vacina e tratamento eficaz para vencer a pandemia, mesmo que tenha a adesão de rigorosos critérios de segurança (Saif, 2020).

A doença provocada pelo covid-19 apresenta como sintomatologia tosse, febre, dispneia, hemoptise e fadiga, já em casos mais graves existe a ocorrência da síndrome do desconforto respiratório (SDRA), falência de múltiplos órgãos e pneumonia. Similarmente, o SARS-CoV-2 também possui a capacidade de infectar diferentes células do corpo humano. Como o destaque, para as células do sistema nervoso central que podem ser infectadas juntamente com sistema respiratório. Estudos comprovam também a existência de sintomas neurológicos em pacientes com COVID-19, tais como: dor de cabeça, anosmia, disgeusia, tontura e consciência prejudicada (Needham, Chou, Coles \& Menon, 2020).

É de fundamental importância compreender as características epidemiológicas e evolutivas tanto do SARS quanto do MERS-CoV, pois, os dois coronavírus estão geneticamente relacionados. A possibilidade de recombinação entre esses dois vírus se ocorrer coinfecção do vírus, o hospedeiro não pode ser descartado devido suas espécies hospedeiras se sobreporem (Zhanget al., 2021).

O sequenciamento de genomas virais se tornou uma ferramenta importante na compreensão e rastreamento da dinâmica das infecções permitindo a reconstrução eficaz da disseminação geográfica viral além de sua estimativa de quantidade epidemiológica como o número de reprodução básica do vírus, taxa de crescimento, tempo de duplicação e padrões de incidência e prevalência da doença (Miller et al., 2020).

Quando o SARS-CoV-2 infecta células que expressam a Enzima Conversora de Angiotensina 2 (ECA-2) e os receptores de superfície TMPRSS2 (células das vias aéreas, células epiteliais alveolares, células endoteliais vasculares e macrófagos no pulmão) o ciclo viral induz a célula a sofrer piroptose que resulta na liberação de padrões moleculares associados ao dano, como por exemplo ATP, ácidos nucleicos e oligômeros de ASC (Tayet al., 2020). Na mucosa nasal e no trato gastrointestinal são sugeridos como via de entrada devido a sua maior expressão de ECA-2. Achados clínicos apontam para perturbações neurológicas em alguns pacientes representados por sintomas de anosmia e ageusia, dentre outros agravos neurológicos. Sendo assim, acredita-se que o SARS-CoV-2 tenha potencial neuroinvasivo (Das, Mukherjee \& Ghoshet, 2020).

Dessa forma, o presente estudo tem como objetivo revisar o material literário existente que descreve as consequências neurológicas associadas ao COVID-19. Pensando em fortalecer as produções de conhecimento científico na área, torna-se fundamental a elaboração de estudos que demonstra clareza ao abordar a temática em questão para assim engrandecer as discussões e respaldar futuros estudos. Busca-se responder a seguinte questão norteadora: Quais as consequências neurológicas que a SARS-CoV-2 pode causar ao sistema nervoso? 


\section{Metodologia}

O presente estudo se refere a uma revisão integrativa de literatura, realizada conforme as etapas: 1) elaboração da questão de pesquisa; 2) levantamento bibliográfico; 3) avaliação dos dados; 4) análise dos dados - divisão, exposição e comparação; 5) apresentação dos resultados (Whittemore \& Knafl., 2005).

O levantamento bibliográfico ocorreu de 25 de setembro a 12 de outubro de 2021. A questão de pesquisa foi estruturada baseada na estratégia PICo (P- População: pacientes com complicações neurológicas; I- Interesse: sequelas causadas pela COVID-19; Co- Contexto: Analisar usuários que tiveram a COVID-19 e desenvolveram complicações neurológicas), o que resultou na questão norteadora: Qual o mecanismo de atuação do SARS-CoV-2 que pode ocasionar sequelas neurológicas?

As bases de dados utilizadas foram Medical Literature Analysis and Retrieval System Online (MEDLINE) e Science Direct.Os descritores utilizados foram: Covid-19 e Nervous System Diseases consultados nos Descritores em Ciências da Saúde (DeCS) e no Medical Subject Headings (MeSH). Realizou-se o cruzamento utilizando-se do operador booleano AND, a saber: "COVID-19" AND "Nervous System Diseases" AND "SARS-CoV-2". Para as bases de dados, adotaram-se as seguintes expressões de busca: (COVID-19) AND (Nervous System Diseases) AND (SARS-CoV-2).

Os critérios de inclusão dos estudos foram: estudos, relatos de casos, séries de casos e revisões que respondessem à questão de pesquisa, foram incluídas publicações entre o ano de 2020 a 2021 para conhecimento das evidências mais atualizadas sobre o conteúdo em questão, foram incluídos artigos nos seguintes idiomas: português, inglês e espanhol. Excluíram-se artigos de reflexão, cartas/comentários ao editor, editoriais de periódicos sem caráter científico.

A pesquisa dos artigos foi realizada de forma independente, pelos pesquisadores. Onde se realizou a leitura dos títulos e resumos, com a escolha criteriosa dos artigos, conforme os critérios de inclusão. Posteriormente, os trabalhos designados na etapa anterior foram lidos na íntegra. Por fim, os estudos foram relidos e analisados, conforme os critérios de elegibilidade, para então, selecionar as publicações que constituíram a amostra final. A busca nas bases de dados resultou em 1.768 publicações, as quais foram selecionadas pelos critérios de elegibilidade, resultando na amostra final de 12 artigos. 
Figura 1 - Fluxograma de seleção dos estudos, segundo o Preferred Reporting Items for Systematic Reviewsand Meta Analyses (PRISMA). Cuité, Paraíba, Brasil, 2021.

\section{PERCUSSO DE SELEÇÃO DOS ESTUDOS}

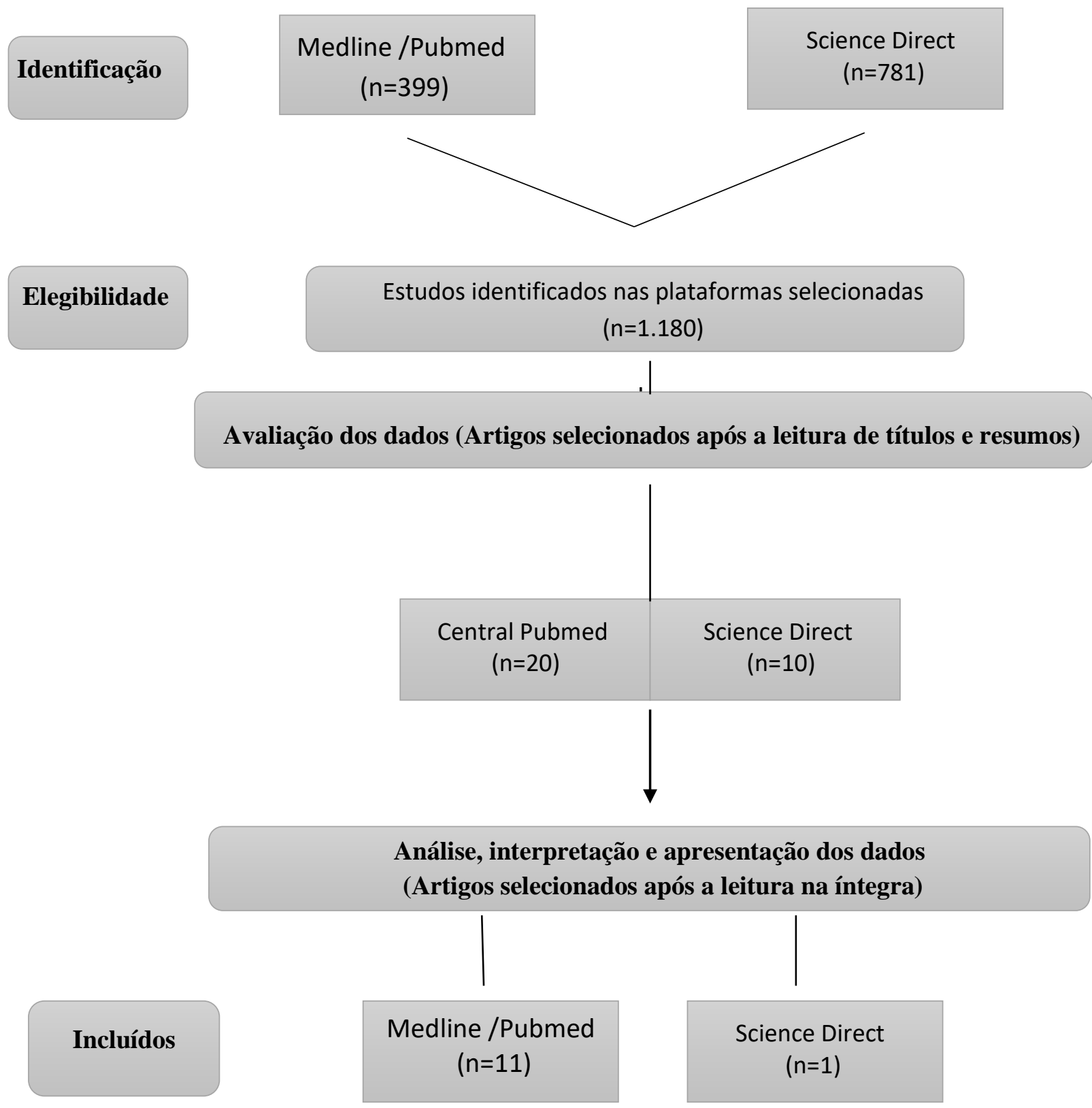

Fonte: Autores.

\section{Resultados e Discussão}

Diante da elegibilidade dos estudos, respeitando os critérios de inclusão e exclusão, foram utilizados 12 artigos para a composição do corpus de análise, selecionando estudos os quais respondiam à pergunta norteadora e que foram publicados entre os anos de 2020 a 2021, considerando o período inicial da pandemia pela SARS-CoV-2 até Outubro de 2021. Os estudos mais atuais foram agrupados no Quadro 1, contendo informações sobre autoria, ano da publicação, título e resultados. 
Objetivando uma coleta de estudos atualizada frente às consequências neurológicas causadas pelo novo coronavírus, os artigos selecionados correspondem a revisões, série de casos e estudos.

Quadro 1. Corpus de análise de pesquisa. Brasil, 2021.

\begin{tabular}{|c|c|c|}
\hline $\begin{array}{c}\text { Autoria e ano de } \\
\text { publicação }\end{array}$ & Título do artigo & Resultados \\
\hline $\begin{array}{l}\text { AGHAGOLI, G.et al., } \\
2020 .\end{array}$ & $\begin{array}{l}\text { Neurological Involvement } \\
\text { in COVID-19 and } \\
\text { Potential Mechanism: A } \\
\text { review }\end{array}$ & $\begin{array}{l}\text { Estudos em animais que examinaram que o receptor de enzima de conversão de } \\
\text { angiotensina-2 é um mediador da lesão neuronal relacionada ao coronavírus e } \\
\text { que a SARS-CoV-2 pode infectar o endotélio cerebrovascular e o parênquima } \\
\text { cerebral em outros estudos, identificaram no cérebro humano pós-morte } \\
\text { coronavírus humano, podendo infectar neurônios e glias. }\end{array}$ \\
\hline GASMI, A. et al.,2020. & $\begin{array}{l}\text { Neurological } \\
\text { Involvements of SARS- } \\
\text { CoV-2 Infection }\end{array}$ & $\begin{array}{l}\text { As diferentes neuropatofisiologias, em pacientes com COVID-19 que apresentam } \\
\text { sintomas do SNC de início precoce, múltiplos e graves ou deteriorização } \\
\text { respiratória rápida devem ser sugestivos para a neuroinvasão viral direta e } \\
\text { alternativas de tratamento apropriadas devem ser consideradas. }\end{array}$ \\
\hline $\begin{array}{l}\text { HARAPAN, B.N.et al., } \\
2021 .\end{array}$ & $\begin{array}{l}\text { Neurological symptoms, } \\
\text { manifestations, and } \\
\text { complications associated } \\
\text { with severe acute } \\
\text { respiratory syndrome } \\
\text { coronavirus } 2 \\
\text { (SARS-CoV-2) and } \\
\text { coronavirus disease } 19 \\
\quad(\text { COVID-19) }\end{array}$ & $\begin{array}{l}\text { A partir do estudo foi realizado a busca das consequências da COVID-19, sendo } \\
\text { elas sintomas neurológicos essenciais e comuns, incluindo disfunções gustativas } \\
\text { e olfatórias, mialgia, cefaléia, estado mental alterado, confusão, delírio e tontura, } \\
\text { são apresentados separadamente nas seções. Além disso, as manifestações } \\
\text { neurológicas e complicações graves como, acidente vascular cerebral, trombose } \\
\text { venosa cerebral (sinusite), convulsões, meningoencefalite, síndrome de Guillain- } \\
\text { Barré, síndrome de Miller Fisher, mielite aguda e síndrome da encefalopatia } \\
\text { reversível posterior (PRES) também são abordadas sistematicamente. }\end{array}$ \\
\hline ARTAL, F.J.C., 2020. & $\begin{array}{l}\text { Neurological } \\
\text { complications of } \\
\text { coronavirus and COVID- } \\
19\end{array}$ & $\begin{array}{l}\text { Sintomas neurológicos foram coletados por pacientes COVID-19 tais como } \\
\text { cefaleia, tontura, mialgia e anosmia, assim como casos de encefalopatia, } \\
\text { encefalite, encefalopatia hemorrágica necrosante, acidente vascular cerebral, } \\
\text { ataques epilépticos, rabomiólise e síndrome de Guillain-Barré, associados á } \\
\text { Infecção por SARS-CoV-2. }\end{array}$ \\
\hline WANG, F. et al.,2020. & $\begin{array}{l}\text { Long-Term Respiratory } \\
\text { and Neurological } \\
\text { Sequelae of COVID-19 }\end{array}$ & $\begin{array}{l}\text { Estudos e relatórios crescentes têm apontado que a infecção por SARS-CoV-2 } \\
\text { envolve o sistema nervoso central (SNC) e o sistema nervoso periférico (SNP) e } \\
\text { direta ou indiretamente danifica os neurônios, levando a sequelas neurológicas de } \\
\text { longo prazo. }\end{array}$ \\
\hline $\begin{array}{l}\text { MAHALAKSHMI, } \\
\text { A.M.et } A L ., 2020 .\end{array}$ & $\begin{array}{l}\text { Does COVID-19 } \\
\text { contribute to development } \\
\text { of neurological disease? }\end{array}$ & $\begin{array}{l}\text { É existente evidências que apontam uma possível transmissão retrógrada do vírus } \\
\text { do epitélio olfatório para regiões do tronco encefálico. }\end{array}$ \\
\hline $\begin{array}{l}\text { WHITTAKER, A. et } \\
\text { al., } 2020 .\end{array}$ & $\begin{array}{l}\text { Neurological } \\
\text { Manifestations of } \\
\text { COVID-19: A systematic } \\
\text { review and current update }\end{array}$ & $\begin{array}{l}\text { Foi realizado e relatado que a cefaléia e a anosmia eram manifestações } \\
\text { neurológicas comuns da SARS-CoV-2. E os sintomas menos comuns eram a } \\
\text { convulsão, acidente vascular cerebral e casos isolados de síndrome de Guillain- } \\
\text { Barré. No entanto, mais pesquisas são necessárias para determinar com precisão } \\
\text { a relação entre os pacientes que desenvolveram sequelas neurológicas, seu estado } \\
\text { clínico e qualquer morbidade mortalidade subsequentes. }\end{array}$ \\
\hline QIN, Y. et al., 2021. & $\begin{array}{l}\text { Long-term microstructure } \\
\text { and cerebral blood flow } \\
\text { changes in patients } \\
\text { recovered from COVID- } \\
19 \text { without neurological } \\
\text { manifestations }\end{array}$ & $\begin{array}{l}\text { Mediante comparação com controles saudáveis, a redução da espessura cortical / } \\
\text { CBF e as mudanças na microestrutura foram mais graves em pacientes com } \\
\text { doença grave do que nos indivíduos com doença leve, especialmente nos } \\
\text { sistemas frontal e límbico. Além do mais, as modificações na microestrutura } \\
\text { cerebral, FSC e parâmetros do trato com os marcadores inflamatórios proteína C } \\
\text { reativa, procalcitonina e interleucina } 6 \text {. }\end{array}$ \\
\hline HAY, M. et al., 2021. & $\begin{array}{l}\text { Serum Neurofilament } \\
\text { Light is Elevated in } \\
\text { COVID-19 Positive } \\
\text { Adults in the ICU and is } \\
\text { Associated with Co- } \\
\text { Morbid Cardiovascular } \\
\text { Disease, Neurological } \\
\text { Complications, and } \\
\text { Acuity of Illness }\end{array}$ & $\begin{array}{l}\text { Um grupo de controle saudável }(n=8) \text { foi examinado para comparação. Os } \\
\text { desfechos e medidas primários foram dados demográficos do sujeito, NfL } \\
\text { (medida de lesão neuronal) sérico, presença e extensão de DCV, diabetes, } \\
\text { pontuação de avaliação de falência orgânica sequencial (SOFA), presença de } \\
\text { complicações neurológicas e dados do painel químico do sangue. }\end{array}$ \\
\hline $\begin{array}{l}\text { FRANKE, C. et al., } \\
2021 .\end{array}$ & $\begin{array}{c}\text { High frequency of } \\
\text { cerebrospinal fluid } \\
\text { autoantibodies in COVID- } \\
19 \text { patients with } \\
\text { neurological symptoms }\end{array}$ & $\begin{array}{l}\text { Foi realizada a utilização de ensaios baseados em células e imunofluorescência } \\
\text { indireta em seções de cérebro murino não fixadas, todos os pacientes } \\
\text { apresentaram autoanticorpos antineuronais no soro ou no LCR. Os antígenos } \\
\text { contam com proteínas intracelulares e de superfície neuronal, como o receptor Y } \\
\text { ou NMDA, mas também vários epítopos específicos indeterminados, que reflete } \\
\text { a ligação ao tecido cerebral observada com certos anticorpos monoclonais } \\
\text { humanos SARSCoV- } 2 \text {. Estes incluíram o endotélio dos vasos, proteínas }\end{array}$ \\
\hline
\end{tabular}




\begin{tabular}{|c|l|l|}
\hline $\begin{array}{c}\text { NEEDHAM, E.J. } \text { et al., } \\
2020 .\end{array}$ & $\begin{array}{l}\text { Neurological Implications } \\
\text { of COVID-19 } \\
\text { Infections }\end{array}$ & $\begin{array}{l}\text { Além das complicações neurológicas diretas da infecção por COVID-19, os } \\
\text { pacientes neurológicos correm o risco de sofrer danos tanto de limitações } \\
\text { estruturais (como o número de leitos de terapia intensiva) quanto da hesitação em } \\
\text { tratar com certos medicamentos necessários devido ao risco de infecção por } \\
\text { COVID-19 nosocomial. }\end{array}$ \\
\hline BEGHI, E. et al., 2020. & $\begin{array}{l}\text { COVID-19 Infection and } \\
\text { Neurological } \\
\text { Complications: Present } \\
\text { Findings and } \\
\text { Future Predictions }\end{array}$ & $\begin{array}{l}\text { O surto causado pelo SARS-CoV-2, um vírus influenza com potencial } \\
\text { neurotrópico, identifica manifestações neurológicas na maioria dos indivíduos } \\
\text { afetados. Distúrbios do sistema nervoso central e periférico estão todos presentes, } \\
\text { enquanto acidente vascular cerebral, ataxia, convulsões e depressão do nível de } \\
\text { consciência são mais comuns em pacientes gravemente afetados. }\end{array}$ \\
\hline
\end{tabular}

Fonte: Autores.

A revisão integrativa possibilitou a análise dos estudos, os quais apontaram diversas consequências neurológicas da SARS-CoV-2, dos mais comuns e frequentes aos sintomas e danos inespecíficos e raros que podem ocorrer devidos a infecção, tanto pelo sistema nervoso central (SCN) como pelo sistema nervoso periférico (SNP). Os sintomas e danos encontrados na pesquisa foram organizados no Quadro 2, a seguir:

Quadro 2. Achados da pesquisa referente ás consequências neurológicas.

\begin{tabular}{c}
\hline Sintomas e danos neurológicos da SARS-CoV-2 \\
\hline Anosmia \\
Ageusia \\
Mialgia \\
Cefaleia \\
Tontura \\
Encefalopatia \\
Encefalite \\
Encefalopatia hemorrágica necrosante \\
Acidente vascular cerebral \\
Convulsões \\
Trombose venosa cerebral (TVC) \\
Meningoencefalite \\
Síndrome de Guillain-Barré
\end{tabular}

Fonte: Autores.

Foram identificados diferentes danos neurológicos associados a infecção por COVID-19, representados no quadro 2, os quais possibilitam discussões sobre como essas consequências podem acontecer e quais os fatores envolvidos. Considera-se que os danos graves, estão mais presentes na população de grupo de risco, idosos e pessoas com comorbidades. No entanto, a COVID-19 também pode acarretar agravos a indivíduos que estão fora do grupo de risco. Os estudos associados ás consequências da SARS-CoV-2 estão bastante direcionados aos cuidados e atenção ao sistema respiratório, o que promove um número mais reduzido de estudos direcionados ao sistema neurológico, contudo, há evidências, séries de casos e estudos que representam danos neurológicos pelo novo coronavírus.

A enzima conversora da angiotensina -2 foi apontada como um mediador da lesão neuronal relacionada com a COVID-19, foi comprovado que o SARS-CoV-2 pode infectar o endotélio cerebrovascular e o parênquima cerebral, o que pode resultar em uma necrose ou apoptose celular. Estudo realizado em post-mortem em humano indicou que o vírus pode infectar neurônios e células da glia, sendo assim, um porte de neurovirulência. Também foram encontrados níveis séricos de citocinas elevados, como resultado da infecção, identificada por uma hiperprodução de citocinas chamada "tempestade de 
citocinas", pode ter grande contribuição relevante de lesão neurológica e pode ser fatal em casos graves e há discussões que considere que o vírus também seja vasculotrópico (Anghagoli, Marin, Katchur, Chaves-Sell, Asaad \& Murphy, 2020).

Acredita-se que, com a infecção do SARS-CoV2 em estado ativo, a circulação sistêmica pode repartir e permitir que o vírus entre em contato com o fluxo sanguíneo cerebral. A comunicação de proteínas de pico SARS-CoV2 e seus receptores endoteliais ACE2 podem elevar a barreira hematoencefálica, endotelial e epitelial nasal comprometida. O processo inflamatório também retarda fluxo sanguíneo, oportunizando a neuroinvasão viral. Logo, a circulação sistêmica poderia então ser um meio de entrada do SARS-CoV2 para o sistema nervoso central (SNC) (Gasmin et al.,2020).

Porém, é considerável uma segunda via neuropatológica que descreve a invasão do cérebro pelo bulbo olfatório e via transcricional nasal superior, sendo a qual a SARS-CoV-2 se propaga transneuronalmente para diversas zonais do cérebro (Harapan \& Yoo, 2020).

Na lesão neuronal relacionada à infecção por SARS-CoV-2 o vírus tem contato com o SNC por meio de duas principais rotas: a via olfativa e a via da barreira hematoencefálica. A migração do vírus para o SNC pode ocorrer diretamente por endocitose com auxílio da elevação de permeabilidade vascular induzida por citocinas inflamatórias e por um mecanismo de transferência indireta chamada "cavalo de tróia". Posteriormente, liga-se ao ACE2, o vírus pode migrar para o SNC diretamente por endocitose com a ajuda de aumento da permeabilidade vascular induzida por citocinas inflamatórias e transferência indireta por meio de um mecanismo de 'cavalo de Tróia'. Depois de se ligar ao seu receptor de membrana, ACE2, o SARS-CoV-2 será engolfado no citosol neuronal. Os RNAs virais acessam a mitocôndria, tornando autofagolisossomos para iniciar a autofagia e / ou apoptose. A microglia e as células imunes produzem citocinas pró-inflamatórias, que dará origem a outras anormalidades na função mitocondrial. A SARS-CoV-2 dentro dos neurônios se combina com os microtúbulos axonais com uma ampliação anterógrada e retrógrada para sinapse e entra no próximo nível de neurônios por mecanismo transsináptico e endocitose. Sendo assim, tanto o vírus quanto a 'tempestade de citocinas' podem fazer a destruição da bainha de mielina dos neurônios, resultando em neuropatologia aguda e crônica (Wang, Kream \& Stefano, 2020).

As hipóteses características da de neuroinvasão e neurovirulência do SARS-CoV-2 é baseadas nas seguintes amostras: - Plausibilidade biológica extrapolada do envolvimento do SNC por outros vírus respiratórios; - Achados de danos neurológicos por coronavírus de outras espécies; - Presença de complicações neurológicas de outros tipos de coronavírus; Amostras de animais com presença de infecção do SNC por coronavírus e a existência de pacientes com COVID-19 que apresentaram manifestações neurológicas (Artal, 2020).

O envolvimento neurológico na COVID-19 pode ser crucial em alguns pacientes. Um subconjunto de pacientes manifesta sintomas neurológicos, como cefaléia, tontura ou um evento cerebrovascular. Relatórios também mostram um início súbito e isolado de anosmia e ageusia, perca do olfato e do paladar, são caracterizados como indicadores iniciais pela infecção e sugerem que o envolvimento neurológico precoce pode ser relevante, para que possa prevenir complicações neurológicas potenciais de longo prazo da infecção por COVID-19. Os efeitos neurológicos presentes na infecção são diversos e podem incluir complicações referentes a infecção viral, resposta imune, doença crítica, terapias relacionadas e recuperação (Anghagoli, Marin, Katchur, Chaves-Sell, Asaad \& Murphy, 2020).

As disfunções gustativas e olfatórias são os sintomas neurológicos súbitos populares da COVID-19 com relação ao envolvimento do SNP e o desenvolvimento desses sintomas encontra-se nos estágios iniciais da doença e, portanto, são considerados marcadores de diagnósticos úteis. Estudos implicam que neurônios sensoriais olfatórios não manifestam ACE 2, o que impede que o SARS-CoV-2 infecte essas células. Porém, as células olfativas do epitélio expressam a ACE 2, o que torna sensíveis a infecção pelo vírus, causando a anosmia (Harapan \& Yoo, 2020).

Em Wuhan, China foi realizado um estudo retrospectivo com 214 pacientes com COVID-19 e descobriu que 36,4\% dos pacientes comportavam manifestações neurológicas da doença, incluindo sintomas relacionados ao SNC (24,8\%), SNP 
$(8,9 \%)$ e músculo esquelético e lesão $(10,7 \%)$. As manifestações neurológicas mais habituais foram á tontura $(16,8 \%)$ e cefaleia (13,1\%). Os pacientes gravemente enfermos eram mais propensos do que os pacientes estáveis afetados a exibir sintomas neurológicos $(45,5 \%)$. Curiosamente, o exame post-mortem de um paciente infectado com SARS-CoV-2, que apresentou confusão e alterações do estado mental, detectou vírus em neurônios do lobo frontal por microscopia eletrônica, apesar do teste de PCR e líquido cefalorraquidiano (LCR) negativo. Partículas virais também foram identificadas em célula endotelial capilar cerebral e observadas ativamente brotando de células endoteliais, ambos fornecendo a primeira evidência direta de SARS-CoV-2 em tecido cerebral humano e implicando uma rota hematogênica direta, potencial para a semeadura do SNC. Este relatório foi seguido por um segundo, identificando e quantificando o vírus SARS-CoV-2 em amostras de tecido cerebral de 8 de 22 pacientes (36\%) que morreram de infecção por COVID-19, estabelecendo mais firmemente o potencial neurotrópico da COV-ID-19 (Anghagoli, Marin, Katchur, Chaves-Sell, Asaad \& Murphy, 2020).

Em outro estudo foi identificado que a mialgia é um sintoma comum observados em pacientes com COVID-19. O predomínio da mialgia é variável diante os estudos. Foi sugestivo que o processo inflamatório generalizado e a tempestade de citocinas, poderiam ser responsáveis pela fisiopatologia da mialgia. Até o momento ainda é incerto a presença de manifestação muscular de COVID-19 devido á inflamação sistêmica não especifica ou invasão do tecido muscular (Harapan \& Yoo, 2020).

Um grupo do Instituto de Neurologia da University College London Queen Square descreveu há pouco tempo cinco grupos significativos de apresentações neurológicas em COVID-19, ou seja, I- encefalopatia com delirium / psicose e nenhuma ressonância magnética ou anormalidades no LCR, II- síndromes inflamatórias do SNC, incluindo encefalite e encefalomielite disseminada aguda, III- acidentes vasculares isquêmicos, IV- distúrbios neurológicos periféricos, incluindo síndrome de Guillain-Barré e plexo braquial, e V distúrbios centrais diversos. Sendo assim, as manifestações neurológicas da SARS-CoV2possuem diferentes manifestações neurológicas e incomuns. As modificações referentes ao estado mental ou a encefalopatia em COVID-19 podem ser danos sistêmicos de respostas imunológicas hiperativas ou a neuroinvasão direta de SARS-CoV2 (Gasmin, et al., 2020).

A encefalopatia é uma síndrome de disfunção cerebral transitória que se apresenta como comprometimento agudo ou subagudo do nível de consciência. O risco de sofrer disfunções do estado mental associado ao COVID-19é elevado em pessoas de idade avançada ou com deterioração cognitiva prévia, como indivíduos que apresentam fatores de risco vascular (hipertensão) e comorbidades precedentes (Artal, 2020).

A inflamação conduzida por citocinas pode acarretar encefalopatia e acidente vascular cerebral, comumente com a neurodegeneração mediada por protease alterada e modificações de neurotransmissor, gerando depressão e ansiedade. Os neurologistas devem estar a par da multiplicidade de aparecimentos dessa infecção viral, que pode surgir mesmo na ausência de sintomas respiratórios proeminentes (Mahalakshmi, et al., 2020).

Outro estudo apontou onze relatos de casos confirmados e outro relato potencial de síndrome de Guillain-Barre (GBS) como sendo uma sequela neurológica significativa de SARS-CoV-2. Dos onze casos relatados em estudo, existe uma variabilidade considerável no início das características da SGB e nos sintomas respiratórios típicos da SARS-CoV-2. Apesar do início inconsistente dos sintomas em relação ao diagnóstico de COVID-19, é reconfortante que a maioria dos achados apresente características clínicas consistentes de fraqueza abundante nos membros inferiores e superiores, e perda dos reflexos tendinosos profundos com anormalidades sensoriais variáveis. Um intervalo de tempo entre a infecção e o desenvolvimento de danos neurológicos é o fenótipo clássico de GBS e foi caracterizado como uma apresentação "pós- infecciosa" (Whittaker, Anson \& Harky, 2020).

Em um estudo de coorte de 100 pacientes hospitalizados na UTI em Tucson, Arizona, entre abril 2020 e Agosto de 2020, 89 testaram positivo para COVID-19 e 11 testaram negativo. Foram identificados níveis séricos de neurofilamento (Nfl) em todos esses pacientes e os comparou entre si com uma coorte de controles saudáveis ( $\mathrm{n}=8$ ). Os níveis médios de Nfl nos 
pacientes com COVID positivo foram significativamente superior em comparação com aqueles observados em pacientes COVID negativo e controles saudáveis e sugerem que os pacientes da UTI COVID-positivo possam ter lesão do SNC. No entanto, nos 50 pacientes positivos para COVID, tinham pelo menos um ou mais diagnósticos como insuficiência cardíaca, hipertensão, doença vascular, doença arterial coronariana, arritmia e hiperlipidemia (Hay, et al., 2021).

Um estudo realizado com pacientes no Departamento de doenças infecciosas do hospital de Tongji, concluiu a atrofia da substância cinzenta, redução generalizada do fluxo sanguíneo cerebral e modificações na microestrutura da substância branca, identificadas na ressonância magnética quantitativa, em pacientes pós COVID-19, o que fomenta evidências de consequências neurológicas na SARS-CoV-2 no período de recuperação a longo prazo (Qin, et al.,2021).

A elevada frequência de autoanticorpos guiados ao cérebro na ausência de outras explicações, sugere uma associação causal com sintomas clínicos, principalmente com hiperexcitabilidade (mioclonia, convulsões). No estudo a neuroimagem deum paciente apresentou uma lesão isquêmica na região da artéria cerebral média direita. Outro detectou edema acentuado do fórnice (Franke, et al.,2020).

A preocupação mais relevante do que a invasão viral direta do SNC é as doenças neurológicas parainfecciosas, como Síndrome de Guillain-Barré, mielite transversa ou encefalomielite disseminada aguda, como vista na epidemia do Zika vírus de 2015-2016. Mas, em uma escala muito mais elevada devido a quantidade de pessoas infectadas, é reconfortante que mesmo com o início propenso a pico de condições parainfecciosas ocorrendo normalmente no período de 4 semanas, não ocorreu nenhum sinal claro de países afetados no início da pandemia (Needham1, Chou, Coles \& Menon,2020).

Alterações do sistema nervoso central e periférico estão presentes, enquanto acidente vascular cerebral, ataxia, convulsões e depressão do nível de consciência são sugestivas em pacientes gravemente afetados. No entanto, complicações pós-infecciosas também são esperadas após COVID-19 (Belghi, Feigin, Caso, Santalucia \& Logroscino, 2020).

Logo, as alterações neurológicas da COVID-19 são existentes, segundos os estudos até o momento, são diversas consequências e fatores que envolvem o sistema neurológico, dos sintomas mais simples aos mais severos. No entanto, diante a pesquisa foi observado que na maioria dos estudos analisados abordaram o desenvolvimento de anosmia e augesia como fatores mais comuns e característicos da infecção viral, mas, mediante os estudos outras consequências também foram abordadas, citadas no quadro 2. Entretanto, são casos raros os quais podem ser manifestados a depender do prognóstico em questão, também é considerável que estudos com abordagem neurológica associada a COVID-19 são bastante recentes. Portanto, medidas de prevenção ainda são muito relevantes, até que o processo de vacinação seja finalizado e alcançado a toda população.

\section{Conclusão}

Assim, a adoção de cuidados específicos com a saúde pode fazer a diferença entre os níveis de comprometimento que é provocado pelas sequelas neurológicas da COVID-19. Além disso, os fatores de ordem cerebral, é um dos principais efeitos fisiológicos que está relacionado à função respiratória, por isso a necessidade da realização de exercícios físicos com acompanhamento de um profissional qualificado. $\mathrm{O}$ ideal é que todas as atividades e práticas realizadas nesses pacientes sejam monitoradas por profissionais que atuam na saúde, devido às limitações individuais de cada indivíduo durante a etapa de recuperação da doença afim de evitar problemas como fadiga, lesão muscular e falta de ar. É perceptível que de acordo com os estudos encontrados, que as manifestações neurológicas encontradas em pacientes com COVID-19 necessitam de assistência para auxiliar os provedores no tratamento, gerenciamento e reconhecimento nas complicações neurológicas potencialmente fatais. 


\section{Referências}

Aghagoli, G. et al. (2021). Neurological involvement in COVID-19 and potential mechanisms: a review. Neurocriticalcare. 34(3), 1062-71. 10.1007/S12028020-01049-4.

Beghi, et al. (2020). COVID-19 infection and neurological complications: presente findings and future predictions. Neuroepidemiology. 54(4), 1-6. $10.1159 / 000508991$.

Carod-Artal, F. J. et al. (2020). Neurological complications of coronavirus and COVID-19. Revista de neurologia. 70(9), 311-22. 10.33588/rn.7009.2020179.

COUTO, M. T., Barbieri, C. L. A. \& Matos, C. C. S. A. (2021). Considerações sobre o impacto da covid-19 na relação indivíduo-sociedade: da hesitação vacinal ao clamor por uma vacina. Saúde e Sociedade. 30. 10.1590/S0104-12902021200450.

Das, G., Mukherjee, N. \& Ghosh, S. (2020). Neurological insights of COVID-19 pandemic. ACS chemical euroscience. 11(9), 1206-09. https://doi.org/10.1021/acschemneuro.0c00201.

Desforges, M. et al. (2020). Human coronaviruses and other respiratory viruses: underestimated opportunistic pathogens of the central nervous system? Viruses. 12(1), 14. https://doi.org/10.3390/v12010014.

Franke, C. et al. (2021). High frequency of cerebrospinal fluid autoantibodies in COVID-19 patients with neurological symptoms. Brain, behavior, andimmunity. 93, 415-9. https://doi.org/0.1016/j.bbi.2020.12.022.

Gasmi, A. et al. (2021). Neurological involvements of SARS-CoV2 infection. Molecular neurobiology. 58(3), 944-49. https://doi.org/10.1007/s12035-02002070-6.

Harapan, B. N. \& Yoo, H. J. (2021). Neurological symptoms, manifestations, and complications associated with severe acute respiratory syndrome coronavirus 2 (SARS-CoV-2) and coronavirus disease 19 (COVID-19). Journal of Neurology. 268(9), 1-13. 10.1007/s00415-021-10406-y.

Hay, M. et al. (2021). Serum neurofilament light is elevated in COVID-19 positive adults in the ICU and is associated with co-morbid cardiovascular disease, neurological complications, and acuity of illness. medRxiv. https://doi.org/10.1101/2021.04.28.21256277.

Helms, J. et al. (2020). Neurologic features in severe SARS-CoV-2 infection. New England Journal of Medicine. 382(23), 2268-70. 10.1056/NEJMc2008597.

Mahalakshmi, A. M. et al. (2021). Does COVID-19 contribute to development of neurological disease? Immunity, inflammation and disease. 9(1), 48-58. 10.1002/iid3.387.

Miller, D. et al. (2020). Full genome viral sequences inform patterns of SARS-CoV-2 spread into and within Israel. Nature communications, 11(1), 1-10. 10.1038/s41467-020-19248-0.

Needham, E. J. et al. (2020). Neurological implications of COVID-19 infections. Neurocriticalcare. 32(3), 667-71. 10.1007/s12028-020-00978-4.

OPAS/OMS BRASIL. (2021). Folha informativa sobre COVID-19. https://www.paho.org/pt/covid19\#datas-noticificacoes.

Qin, Y. et al. (2021). Long-term microstructure and cerebral blood flow changes in patients recovered from COVID-19 without neurological manifestations. The Journal of Clinical Investigation. 131(8). 10.1172/JCI147329.

Saif, L. J. (2020). Vaccines for COVID-19: perspectives, prospects, and challenges based on candidate SARS, MERS, and animal coronavirus vaccines. Euro Med J. 200324. 10.33590/emj/200324.

Tay, M. Z. et al. (2020). The trinity of COVID-19: immunity, inflammation and intervention. Nature Reviews Immunology. 20(6), 363-74. $10.1038 /$ nature 01096 .

Toscano, G. et al. (2020). Guillain-Barré syndrome associated with SARS-CoV-2. New England Journal of Medicine, 382(26), 2574-76. 10.1056/NEJMc2009191.

Wang, F., Kream, R. M. \& Stefano, G. B. (2020). Long-term respiratory and neurological sequelae of COVID-19. Medical science monitor: international medical journal of experimental and clinical research. 26. 10.12659/MSM.928996.

Whittaker, A., Anson, M., Harky, A. (2020). Neurological manifestations of COVID-19: a systematic review and current update. Acta Neurologica Scandinavica. 142(1), 14-22. 10.1111/ane.13266.

Whittermore, R., Knafl, K. (2005). The integrative review: updated methodology. Journal of advanced nursing. 52(5), 546-53. 10.1111/j.13652648.2005.03621.x.

Ye, M., Ren, Y. \& Lv, T. (2020). Encephalitis as a clinical manifestation of COVID-19. Brain, behavior, andimmunity. 88, 945. 10.1016/j.bbi.2020.04.017.

Zhang, A. et al. (2021). Epidemiology and evolution of Middle East respiratory syndrome coronavírus, 2012-2020. Infectious Diseases of Poverty. 10(1), 113. 10.1186/s40249-021-00853-0. 